\title{
Levamisole vs. Autoimmune Leukocytoclastic Vasculitis
}

\author{
Sama Bella ${ }^{\mathrm{a}, \mathrm{c}}$, Thomas Genese ${ }^{\mathrm{a}}$, Hinda Bouali ${ }^{\mathrm{b}}$
}

\begin{abstract}
Levamisole is a veterinary antihelminthic drug that, in the past, was a US Food and Drug Administration-approved drug in humans used to treat various cancers and rheumatoid arthritis because of its immunomodulating effects; however, it is not longer used in the United States since 2000, because of multiple adverse side effects including agranulocytosis and vasculitis. Drug-induced vasculitis is a challenging diagnosis. It is important to recognize that levamisole may not only induce ANCA-associated vasculitis but also other autoimmune markers abnormalities. This case discusses the possibility of levamisole-induced vasculitis and the need to pursue further testing to establish causality in ANCA-associated vasculitis.
\end{abstract}

Keywords: Leukocytoclastic vasculitis; Levamisole; Autoimmune diseases; Drug-induced vasculitis

\section{Introduction}

It is estimated that over $70 \%$ of cocaine consumed in the United States today is contaminated with levamisole [1, 2]. This case demonstrates the need to maintain a high clinical suspicion of levamisole-induced ANCA-associated vasculitis in a patient with characteristic signs and symptoms but also with pre-existing systemic lupus erythematosus (SLE).

\section{Case Report}

A 54-year-old Caucasian-American female with history of SLE on plaquenil $200 \mathrm{mg}$ daily presented to the office with a complaint of a pruritic rash on her face, back, abdomen and all extremities for 1 year. The rash first appeared on her upper extremities and then progressed to her legs. She denied a history of fever, weight loss, alopecia, dry mouth, oral ulcers, painful red eyes, photosensitivity, dysphagia, recurrent miscarriages, or blood clots but did report myalgia and arthralgia on systems

Manuscript submitted November 19, 2017, accepted December 5, 2017

anternal Medicine Department, UHS Wilson Medical Center, Johnson City, NY, USA

bUHS Rheumatology, Johnson City, NY, USA

${ }^{\mathrm{c} C}$ Corresponding Author: Sama Bella, UHS Wilson Medical Center, 33-57 Harrison St, Johnson City, NY, USA. Email: dr.samaalchalabi@gmail.com

doi: https://doi.org/10.14740/jmc2965w review. Further, the patient admitted to crack cocaine use but reported abstinence for the past 18 months.

On examination, vital signs were within normal limits. Skin examination revealed features of retiform purpura on her upper and lower extremities - brightly contoured and irregularly bordered areas of necrosis. A stage II ulcer on the medial surface of her right ankle with some serosanguineous discharge was also discovered. The remainder of her physical examination results was unremarkable.

Investigations revealed leukopenia with a white blood cell count of 2,000 cells $/ \mu \mathrm{L}$, an absolute neutrophil count of 520 cells $/ \mu \mathrm{L}$, and an elevated erythrocyte sedimentation rate of $47 \mathrm{~mm} / \mathrm{h}$. Lupus anticoagulant ratio was $>1: 2$, perinuclear ANCA 1:640, anti-PR3 antibody 18.7, ANA titer 1:320, and anti-SSA were positive $>8.0$, cryoglobulins, anti-cardiolipin antibody, anti-dsDNA, anti-Smith, anti-SSB, anti-RNP, and complement profile were negative or normal. Given her history as well as the clinical findings on examination, a urine drug screen was ordered and was positive for cocaine; subsequent gas chromatography-mass spectrometry was also positive for levamisole. Punch biopsy of the skin from involved areas showed leukocytoclastic vasculitis with fibrin thrombi, angiocentric infiltrates of mixed inflammatory cells, and small vessel thrombosis with multiple fibrin thrombi in the lumen of the vessels (Fig. 1).

Given the patient's history, supportive skin findings, lab profile, and biopsy results, we questioned the prior diagnosis of SLE and instead favored levamisole-induced vasculitis. As

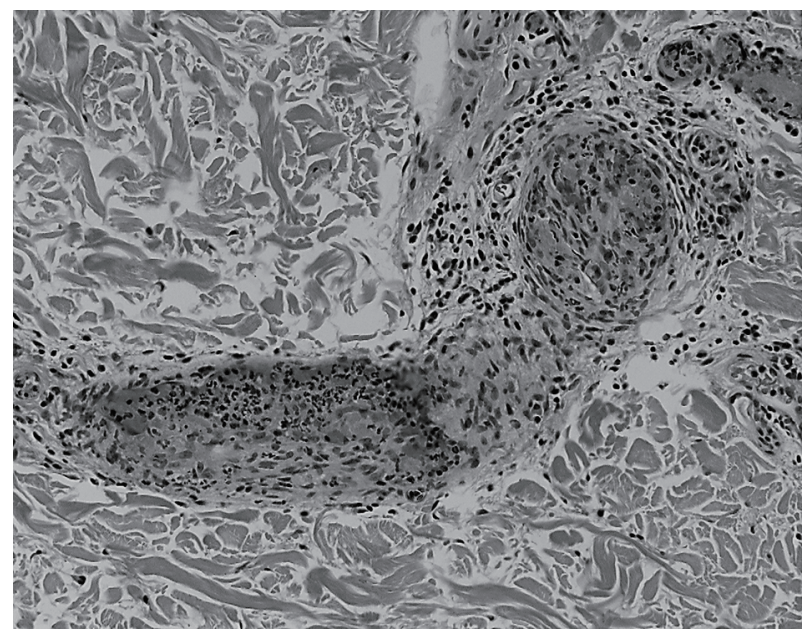

Figure 1. Punch biopsy of the skin from involved areas showed leukocytoclastic vasculitis with fibrin thrombi, angiocentric infiltrates of mixed inflammatory cells, and small vessel thrombosis with multiple fibrin thrombi in the lumen of the vessel. 
such, she was treated with 10 days of oral steroids $20 \mathrm{mg}$ daily, and her skin findings subsequent improved.

\section{Discussion}

Investigational studies have been performed to determine the basis for adulterating cocaine with levamisole [1]. Levamisole may potentially inhibit the degradation of monoamine neurotransmitters dopamine, norepinephrine, and serotonin in the central and peripheral nervous systems leading to reuptake inhibition, prolonged duration of action, and enhanced activity which subsequent may result in enhanced psychotropic effects.

Patients with levamisole-induced vasculitis usually present with a tender, purpuric rash in a retiform or stellate pattern with or without central necrosis involving the extremities; however, $39 \%$ of patients may present without cutaneous findings [3].

The syndrome has a very interesting spectrum of autoantibody findings. High-titer p-ANCA are almost always found (86-100\%), and about $50 \%$ of the cases also have c-ANCA positivity $[4,5]$. The specific antigens responsible for generating these patterns are not yet clearly defined. Antibodies against proteinase- 3 - the autoantibody most commonly associated with a c-ANCA pattern - are present in about $50 \%$ of these patients, while antibodies against myeloperoxidase - the antibody most often responsible for a p-ANCA pattern - are found in almost every case $[1,6]$. In addition, antiphospholipid antibodies and antinuclear antibodies are also often present.

Distinguishing levamisole-induced vasculitis from autoimmune ANCA-associated vasculitis remains a challenge. Detecting levamisole in serum or urine requires gas or liquid chromatography-tandem mass spectrometry which is often not easily accessible. Furthermore, the plasma half-life of levamisole is estimated at only $5.6 \mathrm{~h}$ [7]. Following their series in which only $12.5 \%$ of patients tested positive for levamisole, Gross et al proposed that a positive test for levamisole should not be required to make the diagnosis of levamisole-induced vasculitis in patients with the characteristic clinical presentation [1].

In the absence of clear cocaine abuse history, this case highlights the challenges encountered in differentiating a levamisole-induced versus autoimmune ANCA-associated vasculitis flare. For the former, these patients should be treated and counseled appropriately in an effort to prevent further progres- sion or recurrence of disease.

\section{Conclusions}

Given the high frequency of levamisole adulteration of cocaine, a patient presenting with purpuric rash, neutropenia, and ANCA positivity should be tested for cocaine exposure even in the absence of history of drug use and despite a reported underlying diagnosis of SLE.

\section{References}

1. Gross RL, Brucker J, Bahce-Altuntas A, Abadi MA, Lipoff J, Kotlyar D, Barland P, et al. A novel cutaneous vasculitis syndrome induced by levamisole-contaminated cocaine. Clin Rheumatol. 2011;30(10):1385-1392.

2. Chang A, Osterloh J, Thomas J. Levamisole: a dangerous new cocaine adulterant. Clin Pharmacol Ther. 2010;88(3):408-411.

3. Trimarchi M, Gregorini G, Facchetti F, Morassi ML, Manfredini C, Maroldi R, Nicolai P, et al. Cocaine-induced midline destructive lesions: clinical, radiographic, histopathologic, and serologic features and their differentiation from Wegener granulomatosis. Medicine (Baltimore). 2001;80(6):391-404.

4. Agranulocytosis Associated with Cocaine Use - Four States, March 2008 - November 2009 website. http:// www.cdc.gov/mmwr/preview/mmwrhtml/mm5849a3. htm. Updated December 1, 2009. Accessed October 5, 2017.

5. Rongioletti F, Ghio L, Ginevri F, Bleidl D, Rinaldi S, Edefonti A, Gambini C, et al. Purpura of the ears: a distinctive vasculopathy with circulating autoantibodies complicating long-term treatment with levamisole in children. Br J Dermatol. 1999;140(5):948-951.

6. McGrath MM, Isakova T, Rennke HG, Mottola AM, Laliberte KA, Niles JL. Contaminated cocaine and antineutrophil cytoplasmic antibody-associated disease. Clin J Am Soc Nephrol. 2011;6(12):2799-2805.

7. Kouassi E, Caille G, Lery L, Lariviere L, Vezina M. Novel assay and pharmacokinetics of levamisole and p-hydroxylevamisole in human plasma and urine. Biopharm Drug Dispos. 1986;7(1):71-89. 